\title{
Diagnosis of pulmonary embolism
}

Recent clinical studies have shed new light on the diagnosis of venous thromboembolism. Pulmonary embolism continues to be difficult to diagnose because the definitive test, pulmonary angiography, is complex and not widely available. However, new knowledge about ventilation perfusion lung scanning and non-invasive leg studies can help to reduce the need for pulmonary angiography. Furthermore, the PIOPED study showed that combining a clinical probability assessment of pulmonary embolism with the lung scan probability more accurately establishes the likelihood of pulmonary embolism. This knowledge can be applied widely to aid the clinician in the diagnosis of pulmonary embolism and deep venous thrombosis.

It is now widely accepted that deep vein thrombosis of the lower extremity and pulmonary embolism are two manifestations of the syndrome of venous thromboembolism. Deep vein thrombosis is a progressive process that usually begins in the deep veins of the calf and then propagates directly through the popliteal and into the iliofemoral system. ${ }^{1}$ Thrombosis of the popliteal or more proximal veins is more likely to result in pulmonary embolism than is isolated deep calf vein thrombosis. In most instances the thrombotic process extends directly from the calf upward into the proximal system. On rare occasions the process is isolated in the iliofemoral system, but even in these instances it is usually associated with concurrent separate deep calf vein thrombosis. The pattern of isolated iliofemoral thrombosis is seen most often in individuals who have undergone hip replacement. Venous thrombosis of the lower extremity nearly always precedes pulmonary embolism. There are some reports of clinically significant pulmonary embolism occurring in individuals with thrombosis of the superior vena cava, the innominate and axillary veins. However, these instances are rare and are usually associated either with direct trauma or a hypercoagulable state. Similarly, there are anecdotal reports of pelvic vein thrombosis causing pulmonary embolism, but these instances are also rare and worthy of note in the literature. Pelvic vein thrombosis does occur in individuals following pelvic trauma or malignancy and during pregnancy. However, it is very rare for these thrombi to result in clinically significant pulmonary embolism without the concurrent presence of deep venous thrombosis of the lower extremity. Consequently, the approach to the diagnosis of pulmonary embolism must also include a search for deep venous thrombosis of the lower extremity. This search has been greatly simplified by the emergence of B mode ultrasonography with compression (CUS). ${ }^{23}$ The usefulness of this non-invasive test not only establishes the diagnosis but, when it repeatedly remains negative it identifies a low risk group for subsequent pulmonary embolism of proximal deep vein thrombosis. Consequently, the test can be used to stratify the risk of individuals with suspected pulmonary embolism having subsequent morbid events over the next three months.

Table 1 Major clinical risk factors for venous thromboembolism

\begin{tabular}{|c|c|}
\hline $\begin{array}{l}\text { In-hospital risk factors } \\
\text { for venous } \\
\text { thromboembolism }\end{array}$ & $\begin{array}{l}\text { Trauma (surgery, childbirth, thermal burn) } \\
\text { Immobilisation or other cause of venous statis } \\
\text { Cancer } \\
\text { Older age ( } \geqslant \text { age } 40 \text { ) }\end{array}$ \\
\hline $\begin{array}{l}\text { Risk factors for } \\
\text { outpatient venous } \\
\text { thromboembolism }\end{array}$ & $\begin{array}{l}\text { Male sex } \\
\text { Older age ( } \geqslant \text { age } 60) \\
\text { Cancer } \\
\text { Systemic lupus erythematosis }\end{array}$ \\
\hline
\end{tabular}

\section{Clinical inference and the diagnosis of pulmonary embolism}

The PIOPED study showed that, if the clinician made a prior estimate of the probability of pulmonary embolism based on clinical and laboratory data exclusive of the lung scan results and any other imaging study, then this clinical categorisation could further refine both ends of the spectrum of likelihood for pulmonary embolism. ${ }^{4}$ In this manner a high probability lung scan, when combined with an independent high probability clinical estimate, was associated with a positive angiogram $96 \%$ of the time. At the other end of the spectrum a low probability or near normal lung scan with an independent low clinical probability was associated with a positive pulmonary angiogram less than $5 \%$ of the time. Several caveats are necessary. The clinical estimate must be made independently of knowledge of the lung scan, angiogram, or any non-invasive leg study. The lung scan must be performed and interpreted adequately, and prevalence of pulmonary embolism in the population under study must be at least $20 \%$. The clinical act of suspecting pulmonary embolism and ordering a lung scan seems to assure a prevalence of about $30 \% .{ }^{45} \mathrm{~A}$ common question that arises in this process is the skill of the practitioner at making the estimate of clinical probability. In this regard it is important to note that the PIOPED investigators found that caregivers at different levels of training could make informed and reproducible clinical estimates of the probability of pulmonary embolism. Although validated algorithms for the clinical estimation of probability of pulmonary embolism do not exist, there is general agreement that the clinical estimate should take into account the risk status of the patient, the history and physical findings, and any radiographic and gas exchange abnormalities that are present.

Major risk factors for venous thromboembolism are trauma of any kind including burns and childbirth, but particularly surgical procedures on the lower extremity, immobilisation for more than 48 hours, cancer, and increasing age. These risk factors have been repeatedly identified in studies of patients in hospital. ${ }^{6}$ Major risk factors for idiopathic venous thromboembolism include male sex, increasing age, cancer, and systemic lupus erythematosis (table 1).

It is also useful to consider the clinical syndromes associated with the presentation of pulmonary embolism. The most common syndrome is unexplained acute dyspnoea or tachypnoea, and is seen in patients with pulmonary embolism of all sizes and severity. Shock or syncope, sometimes associated with a right-sided gallop and an increased pulmonary component of the second heart sound, is an uncommon presentation and is usually associated with massive pulmonary embolism. Pulmonary haemorrhage or haemoptysis, often found with an infiltrate localised to the periphery of the lung, is associated with smaller pulmonary emboli that involve segmental or subsequential vessels. It should be noted that this last presentation does not necessarily imply pulmonary infarction. Pulmonary infarction is defined clinically as a long lived infiltrate or fibrotic area on the chest radiograph associated with a previous pulmonary embolism at that location. The short lived hazy infiltrate of localised atelectasis or haemorrhagic infiltration associated with pulmonary embolism does not signify pulmonary infarction. A somewhat unusual presentation is apparent low grade pneumonia with 
Table 2 Factors to consider in estimating clinical probability of pulmonary embolism

\begin{tabular}{cl}
\hline $\begin{array}{c}\text { High } \\
(80-100 \% \text { probable })\end{array}$ & $\begin{array}{l}\text { Risk factor present } \\
\text { Otherwise unexplained dyspnoea, tachypnoea, or } \\
\text { pleuritic pain } \\
\text { Otherwise unexplained radiographic or gas } \\
\text { exchange abnormality }\end{array}$ \\
$\begin{array}{c}\text { Intermediate } \\
(20-79 \% \text { probable })\end{array}$ & Neither high nor low clinical probability \\
Low & $\begin{array}{l}\text { Risk factor not present } \\
\text { Dyspnoea, tachypnoea or pleuritic pain may be } \\
\text { present but explainable by another condition } \\
\text { Radiographic or gas exchange abnormality may be } \\
\text { present but explainable by another condition }\end{array}$ \\
\hline
\end{tabular}

a slowly resolving or unresolving infiltrate, perhaps associated with minimal symptoms such as mild fever, dyspnoea, and tachypnoea. This presentation is seen most often in the elderly and is mistaken for a viral or other atypical pneumonia. In assessing these different clinical presentations it is important to remember that $97 \%$ of patients with pulmonary embolism will have one or more of dyspnoea, tachypnoea ( $\geqslant 20$ breaths $/ \mathrm{min}$ ), or pleuritic pain. ${ }^{8}$ If the clinician remembers these three symptoms and findings when screening for pulmonary embolism, the possibility of pulmonary embolism will rarely be overlooked.

Common radiographic manifestations of pulmonary embolism include focal atelectasis, particularly with elevation of a hemidiaphragm, an ill defined parenchymal density or infiltrate, usually localised to the periphery of the lung, and a small pleural effusion. The common gas exchange abnormality associated with pulmonary embolism is a widened alveolar-arterial oxygen gradient. In most series approximately $80 \%$ of patients with pulmonary embolism will have this finding. ${ }^{9}$

Although there are no validated algorithms for estimating the clinical probability of pulmonary embolism, table 2 gives guidelines for establishing clinical probability. A high probability patient would have a plausible risk factor, one or more of the common screening findings, and a radiographic or gas exchange abnormality. A low probability patient (1-19\% probability) would have no risk factor, clinical symptoms, or findings that were explainable by another disease, and radiographic or gas exchange abnormalities that were also explainable by another condition. Intermediate category patients (20-79\% probability) are those who do not meet the criteria for either the high or low category. Using these guidelines or some reasonable variant, the clinician can estimate clinical probability before examining the lung scan or other diagnostic study.

\section{Lung scan abnormalities}

Recent studies have refined our knowledge of the lung scan abnormalities associated with pulmonary embolism, including the PIOPED database. On lung scans, pulmonary embolism is associated with large perfusion defects, usually in the presence of relatively intact ventilation. ${ }^{4}$ In patients with prior cardiopulmonary disease the specificity of a single large or intermediate perfusion defect seems to be small. However, in people without a history of prior cardiopulmonary disease a single large or intermediate perfusion defect with normal ventilation and a relatively normal chest radiograph is reasonably diagnostic of pulmonary embolism. It also appears that the intermediate perfusion defect (25-75\% of the segment not perfused) is nearly as good as a large perfusion abnormality ( $>75 \%$ of the segment not perfused) in diagnosing pulmonary
Table 3 Effect of prevalence on positive predictive value*

\begin{tabular}{lcccc}
\hline Prevalence of disease (\%) & \multicolumn{4}{c}{ Positive predictive value of test (\%) } \\
\hline 20 & $96 \cdot 1$ & $82 \cdot 6$ & $69 \cdot 2$ & $50 \cdot 0$ \\
10 & $91 \cdot 7$ & $67 \cdot 9$ & $50 \cdot 0$ & $30 \cdot 8$ \\
5 & $83 \cdot 9$ & $50 \cdot 0$ & $32 \cdot 1$ & $17 \cdot 4$ \\
1 & $50 \cdot 0$ & $16 \cdot 1$ & $8 \cdot 3$ & $3 \cdot 9$ \\
$0 \cdot 1$ & $9 \cdot 0$ & $1 \cdot 9$ & $0 \cdot 9$ & $0 \cdot 4$ \\
Sensitivity (\%) & 99 & 95 & 90 & 80 \\
Specificity (\%) & 99 & 95 & 90 & 80
\end{tabular}

* Positive predictive value is the percentage of patients with a positive test result who actually have the disease.

embolism. ${ }^{1011}$ The clinician can amalgamate this information by remembering that, in a patient with a reasonable clinical likelihood of pulmonary embolism (intermediate or high probability category), the presence of two or more perfusion defects that occupy at least $25 \%$ of a segment is acceptable for the diagnosis of pulmonary embolism. This relationship is stronger for patients without prior cardiopulmonary disease but is also reasonably strong for patients with prior cardiopulmonary disease.

\section{Benefit of the clinical probability estimate}

Neither lung scanning nor lower extremity ultrasonography with compression is especially useful in an asymptomatic population at low risk for venous thromboembolism. This apparent discrepancy in the utility of these tests can be illustrated by the effect of prevalence of disease on the positive predictive value of a diagnostic test (table 3). Prevalence does not affect the sensitivity or specificity of a test, but it does affect the positive and negative predictive values. When the prevalence of disease falls well below $20 \%$ the positive predictive value of the diagnostic test falls dramatically. Consequently, when the test is used in a low prevalence population - for example, asymptomatic postoperative patients - the usefulness of the test declines because of a loss of positive predictive value.

When the independent clinical estimate of probability is high or low it seems to move the patient into a high or low prevalence category where the positive or negative predictive value of the imaging test is more useful. In this way the high clinical estimate seems to ensure a sufficiently high prevalence of disease to make the positive predictive value of the imaging test useful. Conversely, the low clinical estimate probably ensures a sufficiently low prevalence of disease to make the negative predictive value of the imaging test useful. These relationships have been validated for the ventilation perfusion lung scan in the diagnosis of pulmonary embolism, but probably also hold for duplex ultrasonography and impedance plethysmography in the diagnosis of deep venous thrombosis. ${ }^{12}$

\section{Integrated approach to diagnosis}

Deep venous thrombosis precedes pulmonary embolism. Approximately $50 \%$ of those with known pulmonary embolism will have diagnosable deep venous thrombosis of the lower extremity at the same time. Patients with pulmonary embolism or suspected pulmonary embolism who have a repeatedly negative test of their lower extremity venous system appear to be at low risk for subsequent morbidity from venous thromboembolism. Consequently, the integrated approach to the diagnosis of venous thromboembolism relies both on the clinical acumen of the clinician and on the use of the two major screening tests - that is, ventilation perfusion lung scanning and B mode ultrasonography with compression of the lower extremity. An 


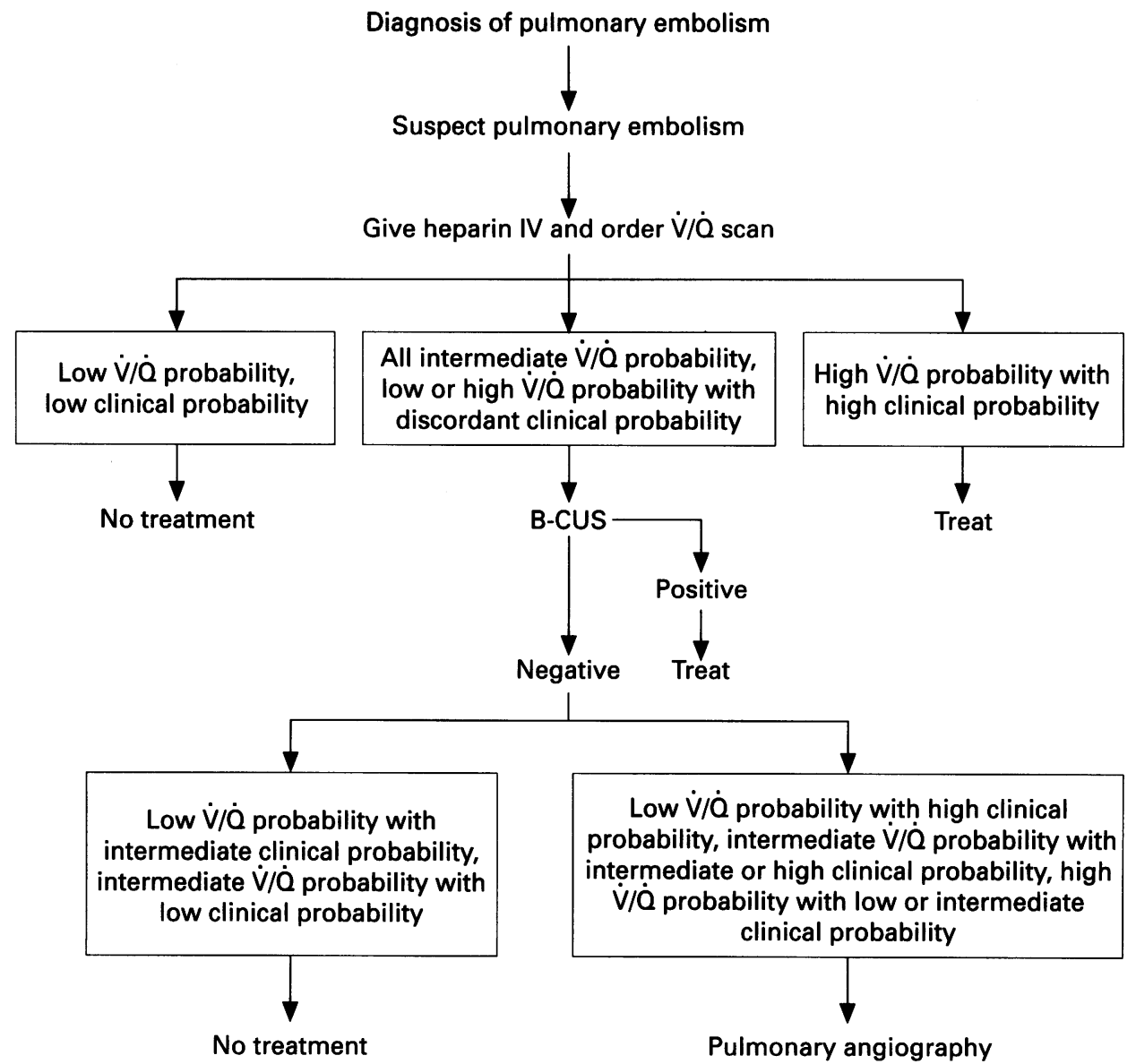

\begin{abstract}
Integrated diagnostic approach to pulmonary embolism using clinical probability, ventilation/perfusion $(\dot{V} / \dot{Q})$ lung scanning, ultrasonography with compression (B-CUS), and pulmonary arteriography. This approach can significantly reduce the need for pulmonary angiography in patients with suspected pulmonary embolism from approximately two in three cases to one in three.
\end{abstract}

algorithm for the use of the combined approach is given in the figure. This algorithm identifies patients with a high probability of pulmonary embolism (high $\dot{\mathrm{V}} / \mathbf{Q}$ probability, high clinical probability) who would be candidates for long term treatment and would not need pulmonary angiography, and those with a low probability (low $\dot{\mathrm{V} / Q}$ Q probability or near normal $\dot{\mathrm{V}} / \mathbf{Q}$ pattern, low clinical probability) in whom it would be appropriate to withhold treatment. If the clinician continued to be concerned about the possibility of venous thromboembolism in individuals with a low probability rating, follow up surveillance with the non-invasive leg study is appropriate. All other combinations of scan and clinical probability categories would undergo B mode ultrasonography with compression. Anyone with a positive leg study would receive long term treatment. Thus, patients with negative leg scans and combinations of low clinical and intermediate $\dot{V} / \mathbf{Q}$ probability or intermediate clinical and low $\dot{V} / \dot{Q}$ probability would not be candidates for treatment. Patients in other categories would proceed to pulmonary angiography. An alternative approach in these undiagnosed patients would be to follow them with 2-3 non-invasive leg studies over 10-14 days and treat only those who become positive. ${ }^{513}$ A similar approach using clinical inference and non-invasive leg studies can probably be applied to patients with suspected deep venous thrombosis of the lower extremity. ${ }^{12}$

Division of Pulmonology and

THOMAS M HYERS

Pulmonary Occupational Medicine,

St Louis University Health Sciences Center,

St Louis,

Missouri 63110-0250,

USA
1 Cogo A, Lensing AWA, Prandoni P, Hirsh J. Distribution of thrombosis in patients with symptomatic deep vein thrombosis. Arch Intern Med 1993, 153:2777-80.

2 Heijboer H, Büller HR, Lensing AWA. A comparison of real-time compression ultrasonography with impedence plethysmography for the diagnosis of deep-vein thrombosis in symptomatic patients. $N$ Engl $₹ \mathrm{Med}$ 1993;3129:1365-9.

3 Ginsberg JS, Wells PS, Hirsh J, Panju AA, Patel MA, Malone DE, et al. Reevaluation of the sensitivity of impedance plethysmography for the detection of proximal deep vein thrombosis. Arch Intern Med 1994;154 1930-3.

4 A Collaborative Study by the PIOPED investigators. Value of the ventilation perfusion scan in acute pulmonary embolism - results of the prospective investigation of pulmonary embolism diagnosis (PIOPED). $\mathfrak{f} A M A 1990$ 263:2753-9.

5 Stein PD, Hull RD, Saltzman HA. Strategy for diagnosis of patients with suspected acute pulmonary embolism. Chest 1993;103:1533-9.

6 Stein PD, Terrin ML, Hales CA, Palevsky HI, Saltzman HA, Thompson BT, et al. Clinical, laboratory, roentgenographic and electrocardiographic findings in patients with acute pulmonary embolism and no-prexisting cardiac or pulmonary disease. Chest 1991;100:598-603.

7 Cogo A, Bernardi E, Prandoni P, Girolami B, Noventa F, Simioni P, et al. Acquired risk factors for deep vein thrombosis in symptomatic patients. Arch Intern Med 1994;154:164-8.

8 Stein PD, Henry JW, Gottschalk A. The addition of clinical assessment to stratification according to prior cardiopulmonary disease further optimizes the interpretation of ventilation/perfusion lung scans in pulmonary embolism. Chest 1993;104:1472-6.

9 Stein PD, Athanasoulis C, Greenspan RH, Henry JW. Relation of plain chest radiographic findings to pulmonary arterial pressure and arterial blood oxygen levels in patients with acute pulmonary embolism. Am $\mathcal{f}$ Cardiol 1992;69:394-6.

10 Stein PD, Henry JW, Gottschalk A. Mismatched vascular defects: an easy alternative to mismatched segmental equivalent defects for the inalternative to mismatched segmental equivalent defects for the in-
terpretation of ventilation/perfusion lung scans in pulmonary embolism. terpretation of ventilation

11 Stein PD, Gottschalk A, Henry JW, Shivkumar K. Stratification of patients according to prior cardiopulmonary disease and probability assessment based upon the number of mismatched segmental equivalent perfusion defects: approaches to strengthen the diagnostic value of ventilation perfusion lung scans in acute pulmonary embolism. Chest 1993;104: 1461-7.

12 Wheeler HB, Hirsh J, Wells P, Anderson Jr FA. Diagnostic tests for deep vein thrombosis. Clinical usefulness depends on probability of disease. Arch Intern Med 1994;154:1921-8.

13 Hull RD, Raskob GE, Coates G, Panju AA, Gill GJ. A new noninvasive management strategy for patients with suspected pulmonary embolism. Arch Intern Med 1989;149:2549-55. 